\title{
IMPACTO DO SISTEMA DE MONITORIZAÇÃO CONTÍNUA DA GLICOSE EM PACIENTES DIABÉTICOS
}

\author{
Frederico F. R. Maia*, Levimar R. Araújo
}

Trabalho realizado no Departamento de Ciências Fisiológicas, Disciplina de Fisiologia e Clínica de Endocrinologia e Metabologia do Hospital Universitário São José, Faculdade de Ciências Médicas de Minas Gerais, Belo Horizonte, MG

\author{
*Correspondência: \\ R. Nunes Vieira, 299 \\ ap.702 \\ CEP: $30.350-120$ \\ Belo Horizonte, MG \\ Tel: (31) 3296-3345 \\ fredfrm@hotmail.com \\ frederico@diabetes.med.br
}

\begin{abstract}
RESUMO
OвjetIvo. Avaliar a acurácia da CGMS em pacientes diabéticos, bem como seu impacto no controle glicêmico/metabólico e possíveis complicações do método.

Métodos. Foram estudados 53 pacientes (47,2\%M/52,8\%F), idade: 29,74 I6,38 anos, predomínio de DMI (86,8\%), submetidos à monitorização contínua da glicose (CGM) (Medtronic; Northridge, CA) por $72 \mathrm{~h}$. Foram analisados: glicemia capilar (GC) média e pelo sensor CGMS, coeficiente de correlação, excursões glicêmicas (CGMS versus GC), hiperglicemia pós-prandial (HPP) e hipoglicemia assintomática; complicações e conduta após a CGM. Os níveis de Al c foram medidos antes (um mês) e três meses após a CGM.

Resultados. A GC média durante a CGM foi 191,3 $\pm 45,6 \mathrm{mg} / \mathrm{dl}$ versus $192,5 \pm 43,9 \mathrm{mg} / \mathrm{dl}$ detectada pelo sensor, com correlação altamente significativa $(p=0,001)$. 0 coeficiente de correlação foi de $0,87 \pm 0,16(V R>0,79)$. O CGMS detectou mais excursões glicêmicas em relação à $G C(p=0,001)$. HPP foi evidenciada em $77,3 \%$ dos casos e a hipoglicemia noturna assintomática em $54 \%$. Observou-se redução significante da Alc três meses após a CGM $(p=0,00 \mathrm{I})$. Não houve complicações durante a CGM em $89,8 \%$ dos exames.
\end{abstract}

ConcLusão. A CGMS mostrou-se método seguro, com baixo índice de complicações e alta acurácia nos valores glicêmicos em relação à glicemia capilar. Verificou-se alta eficácia da CGMS na deteç̧ão de excursões glicêmicas, hipoglicemia noturna assintomática e HPP, com importante impacto na redução da Alc após três meses nesse grupo de pacientes.

UnITERMOs: CGMS. Glicemia capilar. Diabetes mellitus.

\section{INTRODUÇÃo}

A glicemia capilar (GC) no monitoramento do diabetes mellitus (DM) é um grande avanço, realizada com amostras coletadas em ponta de dedo, imprescindível ao controle do diabetes mellitus tipo I (DMI) e de substancial importância na avaliação do paciente diabético tipo 2 (DM2)'. A dor dificulta a realização da GC em "ponta de dedo" em função das inúmeras terminações nervosas nesse local2,3. O uso de locais alternativos para monitorização tem sido recentemente estudado, visando um maior conforto para o paciente ${ }^{1,3}$.

A monitorização do DM pela GC fornece apenas dados intermitentes do controle metabólico, impedindo uma visão completa do perfil glicêmico do paciente!. Com o sistema de monitorização contínua da glicose (CGMS), tem sido possível avaliar com exatidão as variações glicêmicas ao longo do dia, com intervenção terapêutica mais eficaz e redução da glico-hemoglobina $(A \mid c)^{4,5}$.

Uma das principais dúvidas da eficácia do método está relacionada à incerteza glicêmica, em períodos de normo, hipo e hiperglicemia $a^{6,7}$. Apesar de níveis adequados de glico-hemoglobina (Alc), crianças e adolescentes com DMI apresentam freqüentes eventos de hipoglicemia e hiperglicemia pós-prandial (HPP), não evidenciados pela GC de rotina6.
No Brasil, não há informações precisas sobre a acurácia desse método e seu impacto no controle glicêmico em pacientes diabéticos. Esse estudo buscou avaliar a acurácia da CGMS, bem como seu impacto no controle metabólico (redução da Alc) e possíveis complicações do método em pacientes diabéticos.

\section{Métodos \\ Pacientes}

Trata-se de estudo retrospectivo, com base em revisão de prontuários de 53 pacientes com diabetes atendidos no Instituto Avançado em Diabetes e Endocrinologia (MG), entre setembro de 2003 e julho de 2005. Todos os pacientes foram submetidos ao CGMS (Medtronic MiniMed; Northridge,CA) por 72h; com tempo médio de DM de $12,8 \pm 9,6$ anos, sendo $47,2 \%$ do sexo masculino e $52,8 \%$ do sexo feminino. A idade variou de 3 a 73 anos, com média de 29,74 16,38 anos. Não houve restrição quanto a idade, sexo ou tempo de diabetes. Houve predomínio do DMI, presente em $86,8 \%$ dos casos.

\section{Sensor de glicose (CGMS)}

A monitorização contínua da glicose (CGM) foi realizada pelo monitor Medtronic/Northridge,CA, primeiro aparelho aprovado pelo 


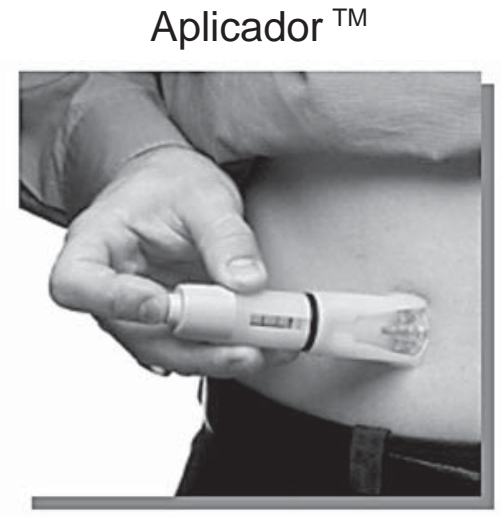

Software

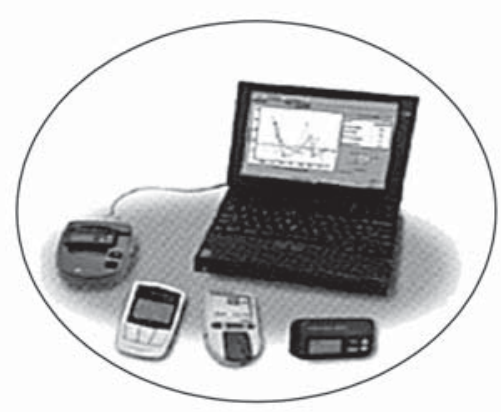

Monitor

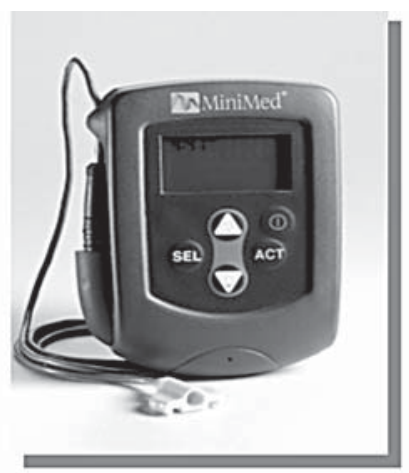

Com-station ${ }^{\mathrm{TM}}$

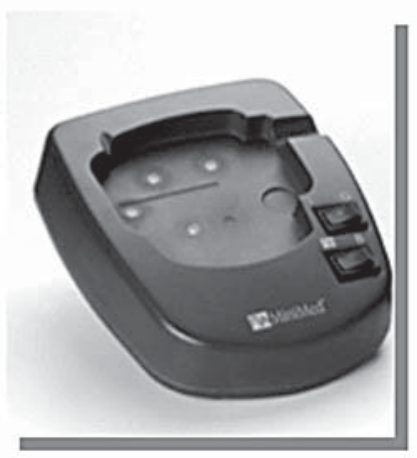

FDA (Food and Drug Administration, EUA). O equipamento é constituído por um sensor, um aplicador, um monitor e um com-station (estação de transferência de dados para o computador), como mostrado na Figura I.

A glicose é mensurada com base na reação eletroquímica da enzima glicose-oxidase presente no sensor com a glicose do fluido intersticial. Os valores variam entre 40 e 400 mg/dl e são captados a cada 10 segundos, com o registro da média desses valores a cada 5 minutos, num total de 288 medidas/dia e 864 medidas durante 72h. Sessenta minutos após a instalação, a corrente elétrica em nanoamperes gerada é convertida em glicose, após a inclusão de um valor de GC no monitor. Ao final do registro, o sensor é descartado e os dados armazenados são transferidos ao software, para análise dos dados.

\section{Procedimento}

Todos os pacientes foram submetidos previamente às orientações básicas de funcionamento do monitor, pelo mesmo examinador, e preenchimento detalhado do "diário do paciente". Os pacientes foram orientados a realizar e incluir um mínimo de quatro valores de GC no monitor por dia. A monitorização foi realizada pelos próprios pacientes, com glicosímetro digital (Accu-Chek Active; Roche Diagnosis), lancetador Accu-Chek Softclix ${ }^{\circledR}$ Pro e respectivas lancetas. A primeira GC foi realizada após 60 minutos do início da CGM.

A instalação do equipamento (CGMS) foi realizada sempre pelo mesmo examinador, com técnica adequada e inserção do sensor em região subcutânea abdominal, com auxílio de um aplicador específico. Os passos para execução do procedimento foram detaIhados na Tabela I.

As variáveis analisadas foram: glicemia capilar média (GCM); glicose pelo sensor CGMS; coeficiente de correlação; mediana da diferença percentual absoluta (MAD\%); número de leituras; excursões glicêmicas pelo sensor CGMS e pela GC episódica; tempo de hipo, normo e hiperglicemia durante o registro de CGMS; presença de HPP e hipoglicemia noturna assintomática; níveis de Alc antes (um mês) e três meses após a CGM; complicações (trauma, infecção local, sangramento, aversão psicológica, inoperabilidade técnica, desconexão, outros "alarmes"); abandono e mudança de conduta após a CGMS.

O coeficiente de correlação entre GC / glicose registrada pelo sensor e o MAD\% basearam-se na análise do programa Medtronic, definidos como ideal com valores de $>0,79 \%$ e $<28 \%$, respectivamente. A MAD\% foi determinada pela mediana da diferença entre o valor absoluto da glicose captada pelo sensor menos o valor de glicose 


\section{Tabela I - Instalação do equipamento de monitorização subcutânea contínua da glicose (CGMS) em pacientes diabéticos, Inade, Belo Horizonte/MG. Seqüência de Eventos}

I. Mostrar o monitor, explicando as teclas: SEL - ACT - $\uparrow$ - $\downarrow$;

2. Mostrar como inserir as glicemias capilares e os eventos;

3. Verificar se o monitor está limpo/ zerado;

4. Codificar a identificação do paciente - desligar o monitor;

5. Preparar o aplicador: adaptar o sensor (retirar o adesivo da frente, travar o aplicador para trás e manter a proteção externa da agulha). O sensor não deve ser congelado. Retirar o sensor da refrigeração cerca de 5 minutos antes;

6. Cortar o adesivo e deixar próximo;

7. Verificar o local em que o sensor será inserido e proceder a assepsia;

8. Retirar a proteção externa da agulha, adaptar o aplicador na pele e acionar o botão injetor;

9. Apoiar com dois dedos o sensor que foi inserido na pele e retirar o aplicador delicadamente; ainda com o apoio dos dois dedos, retirar a agulha-guia e o adesivo de trás;

10. Adaptar o cabo do monitor no sensor até ouvir um "clic" e ligar o monitor;

II. Colocar um adesivo (em torno de $10 \mathrm{~cm} \times 10 \mathrm{~cm}$ ) poroso sobre o sensor;

12. Adaptar o monitor com o clip mais a capa de couro, na cintura. Deixá-lo o mais confortável possível.

captado pelo glicosímetro. O número de leituras pelo sensor foi considerado significativo quando acima de $80 \%$ (> 640 leituras / 72h).

As excursões glicêmicas identificadas pela GC foram baseadas na informação ("diário") do paciente e análise do glicosímetro e comparadas com os registros do sensor CGMS, sendo considerado glicemia abaixo de $70 \mathrm{mg} / \mathrm{dl}$ (hipoglicemia) e acima de $180 \mathrm{mg} / \mathrm{dl}$ (hiperglicemia). O tempo de hipo, normo e hiperglicemia foi registrado em horas / porcentagem, para efeito de comparação.

A HPP foi definida para glicemia superior a $140 \mathrm{mg} / \mathrm{dl}$ duas horas após o almoço. A hipoglicemia noturna assintomática foi definida por glicemia $<70 \mathrm{mg} / \mathrm{dl}$ entre $23 \mathrm{~h}$ e $7 \mathrm{~h}$, na ausência de sintomas. A correlação sensor/GC, bem como a sensibilidade e especificidade, foram avaliadas durante normo, hipo e hiperglicemia, através do teste t de Student.

Os níveis de Al c foram determinados antes (um mês) e três meses após o CGMS. Os valores de Alc foram estabelecidos pelo método HPLC, com valores de referência entre 4,3\% e 6,9\%.

A detecção de complicações foi baseada na informação do paciente, incluindo trauma, infecção, sangramento, aversão psicológica, inoperabilidade técnica, desconexão e outros "alarmes". A tolerância dos pacientes ao CGMS baseou-se na taxa de abandono e necessidade de interrupção do método.

\section{Estatística}

Os dados foram coletados em uma ficha padrão e analisados pelo software Minitab, através do teste do Qui-quadrado, teste t de Student e teste de regressão. Foi considerado um $p<0,05$ para valor estatisticamente significante.

\section{Resultados}

A GC média durante a CGMS foi de 191,3 $\pm 45,6 \mathrm{mg} / \mathrm{dl}$ verus 192,5 $\pm 43,9 \mathrm{mg} / \mathrm{dl}$ pelo sensor CGMS. Não houve diferença significante entre os valores de glicose captados pelo CGMS e GC $(p=0,79 ; T=-0,26)$.
O número de leituras pelo sensor CGMS foi de $823,7 \pm 237,2$ (VR>680), com duração de 69,5 $\pm 15,7 \mathrm{~h}$ de exame. $O$ coeficiente de correlação foi de 0,87 $\pm 0,16(V R>0,79)$, com valores ideais em 90,5\% dos casos. Quanto à MAD\%, observou-se valor médio de $14,2 \pm 5,4 \%$ (VR<28\%), com valor eficaz em 96,2\% dos casos.

Quanto às excursões glicêmicas, a CGMS mostrou-se mais eficaz na detecção de excursões glicêmicas em relação à $G C(T=5,95 ; p=$ $0,001)$.

Quanto à HPP, foi evidenciada em 77,3\% dos casos, com valor médio de $173, I \pm 50,5 \mathrm{mg} / \mathrm{dl}$. A hipoglicemia noturna assintomática foi detectada em $54 \%$ dos casos. O fenômeno do alvorecer foi observado em $11,3 \%$ dos examinados.

Durante as $72 \mathrm{~h}$ de CGMS, os pacientes permaneceram $45,5 \%$ do tempo em hiperglicemia, 8,7\% em hipoglicemia e 45,8\% em normoglicemia, em média.

A avaliação do controle metabólico após três meses da CGMS evidenciou redução significante da Alc nesses pacientes (8,78 \pm ।,4 verus $7,50 \pm 1,3 ; p=0,001$ ).

A correlação entre o sensor e GC durante hipoglicemia assintomática mostrou-se não significante $(p=0, \mid I)$ versus durante hiperglicemia $(p=0,002)$ e normoglicemia $(p=0,05)$ (Fig. 2). O sensor CGMS apresentou baixa sensibilidade $(75,1 \%)$ e alta especificidade $(95,5 \%)$ para hipoglicemia assintomática versus durante hiper e normoglicemia (Tabela 2).

Em 89,8\% dos exames, não foram observadas complicações durante a CGM. O sinal de desconexão foi o mais comum nesses casos ( I I,7\%). Não houve registro de trauma, reação alérgica, infecção local ou sangramento. Detectou-se aversão psicológica e abandono em um paciente com DM2 (> 60 anos). Dificuldade operacional foi relatada em $5 \%$ dos pacientes.

A CGMS promoveu mudança de conduta em $100 \%$ dos pacientes, incluindo ajuste de dose de insulina, modificação do tipo de insulina utilizada, início de contagem de carboidratos e atividade física, além de acompanhamento psicológico em casos especíícos. 
MAIA FFR ET AL.

Figura 2 - Correlação dos valores de glicose detectados pelo sensor do sistema de monitorização contínua da glicemia (CGMS) versus glicemia capilar em pacientes diabéticos em estado hipoglicemia $(<70 \mathrm{mg} / \mathrm{dl})(\mathrm{p}=0, \mathrm{II})$

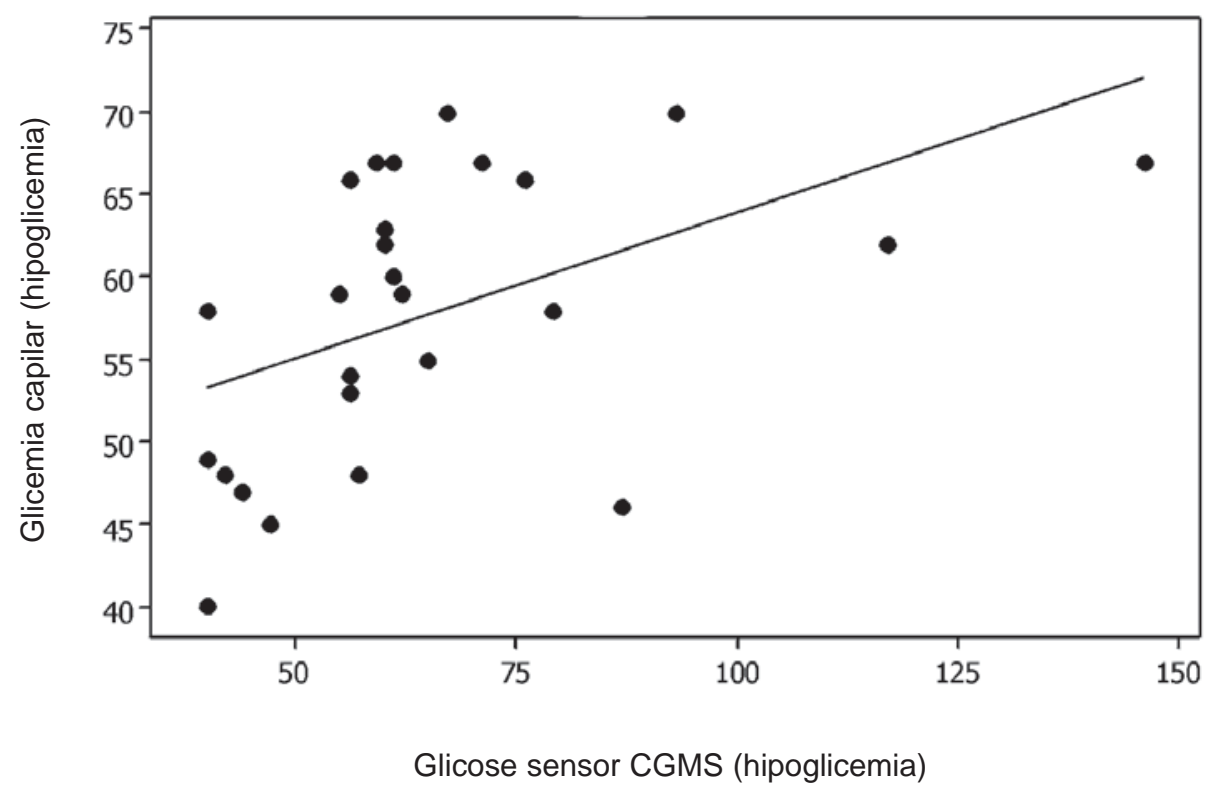

Tabela 2 - Sensibilidade e especificidade da monitorização subcutânea contínua da glicose (CGMS) na detecção de hipo, hiper e normoglicemia, em pacientes diabéticos

\begin{tabular}{lccc}
\hline Estadoglicêmico & $\begin{array}{c}\text { Sensibilidade } \\
(\%)\end{array}$ & $\begin{array}{c}\text { Especificidade } \\
(\%)\end{array}$ & $\begin{array}{c}\text { Valor } \\
\text { Hipoglicemia }\end{array}$ \\
\cline { 2 - 2 } & 79,1 & 97,5 & $\frac{\mathrm{P}}{0,11}$ \\
Normoglicemia & 94,5 & 93,2 & 0,05 \\
Hiperglicemia & 96,8 & 95,4 & 0,002 \\
\hline
\end{tabular}

\section{Dıscussão}

Verificou-se um alto índice de correlação entre as medidas captadas pelo sensor CGMS e os valores da GC associados à tolerância satisfatória pelos pacientes e baixo índice de complicações durante 0 procedimento.

Quanto à acurácia da CGMS, observou-se alto índice de correlação, o que é corroborado pela literatura ${ }^{4,5,8-10}$. Em estudo recente com crianças e adolescentes, foi observada maior eficácia da CGMS em relação a GC 8x/dia na identificação de HPP, porém, com impacto semelhante na redução da $\mathrm{Al}$ c. Os autores reforçam o fato de apenas $10 \%$ dos pacientes terem completado as oito pontas de dedo durante três dias".

Ao analisar a correlação dos valores da GC e do sensor CGMS em 2 I pacientes com DMI (8x/dia), Djakoure-Platonoff et al. obtiveram um coeficiente de correlação de 0,92, com significância em 93\% dos casos. Concluíram que a CGMS 72h oferece alta acurácia nas medidas obtidas, sendo método de eleição para determinar o perfil glicêmico do diabético ${ }^{9}$, corroborados por Guerci et al. ${ }^{10}$.

Quanto ao perfil metabólico dos pacientes após a CGM, diversos estudos evidenciam a eficácia desse método na redução da $A I^{12}$, 13. Estudo com 12 adolescentes (Alc>8\%), com DMI, submetidos à CGM 72h, permitiu identificar excursões glicêmicas não detectadas pela GC em 100\% dos casos, HPP em 10/I2 e hipoglicemia noturna em $33 \%$, com redução significativa das excursões glicêmicas e da Alc $(p<0,05)$ após dois meses ${ }^{14}$.

Em relação às excursões glicêmicas, observamos alta eficácia da CGMS em relação à GC episódica na casuística apresentada $(p<0,01)$, o que vem sendo comprovado pela literatura ${ }^{15-20}$. Dados de 91 pacientes (DMI), submetidos à CGMS $72 \mathrm{~h}$ e com excursões glicêmicas induzidas, mostraram maior acurácia do sensor par níveis mais elevados 
de glicose, sendo menor a sensibilidade para hipoglicemia $(p<0,05)$. Tal evidência sugere o uso da CGMS para fins de redução da Alc, sendo seu impacto sobre as crises de hipoglicemia ainda indefinidos ${ }^{15}$.

Em relação à identificação de episódios de hipoglicemia, alguns estudos vêm comprovando a acurácia da CGMS para detecção de hipoglicemias ${ }^{16,19,20}$, enquanto outros demonstram pouca eficácia desse método para tal finalidade ${ }^{17,18}$. Boland et al. observaram superioridade da CGMS na detecção de HPP (90\%) e de hipoglicemia assintomática (70\%), considerando a CGM exame de grande importância para otimizar o controle metabólico de crianças com DMI ${ }^{19}$.

Guerci et al. evidenciaram sensibilidade de 33\% do sensor para detecção de hipoglicemia ${ }^{10}$. Dados recentes comprovam uma menor eficácia da CGMS para detectar níveis glicêmicos reduzidos, sendo sua principal indicação a melhora do controle metabólico nesses pacientes ${ }^{11,20,21}$. Jeha et al. demonstraram baixa sensibilidade do sensor CGMS para hipoglicemia (70\%), com predomínio de hipoglicemia assintomática no período noturno e maior eficácia na detecção de HPP em crianças com DMI menores que 6 anos $^{21}$, o que é comprovado pelos dados deste estudo (sensibilidade para hipoglicemia de $75 \%$ ) e em crianças e adolescentes entre 6 e 20 anos $^{22}$.

Chico et al. compararam a eficácia da CGMS na identificação de hipoglicemia noturna assintomática em 70 pacientes com DM em relação à CG. A CGMS detectou hipoglicemia assintomática em 62,5\% dos DMI e $46,6 \%$ dos pacientes com DM2, sendo que $73,7 \%$ dos eventos ocorreram no período noturno, sem diferença quanto à redução da $\mathrm{Al}$ c nos dois grupos ${ }^{20}$.

Quanto às complicações, a CGM mostrou-se método de ótima tolerância. Não houve registro de trauma local, infeção ou sangramento.

A mudança de conduta terapêutica foi instituída em todos os pacientes neste estudo, o que vem sendo corroborado pela literatura $11-15,19,20$.

\section{ConClusão}

Este estudo evidenciou a eficácia da CGMS na detecção de excursões glicêmicas, hiperglicemia pós-prandial, na redução da Alc e ajuste terapêutico em pacientes diabéticos. A CGMS mostrou ser método seguro, bem tolerado, com baixa sensibilidade para estados hipoglicêmicos, podendo superestimar os índices de hipoglicemia assintomática em pacientes diabéticos.

\section{Conflito de interesse: não há.}

\section{SUMMARY}

Metabolic / glycemic control accuracy, efFect and COMPLICATIONS OF THE CONTINUOUS GLUCOSE MONITORING SYSTEM IN DIABETIC PATIENTS

OBJETIVE. To evaluate the accuracy, safety and complications of the continuous glucose monitoring system (CGMS) in diabetic patients (DM). The impact of this system on metabolic/glycemic control is still under discussion.

Methods. The 53 patients studied (47.2\% male / 52.8\% female), average age: $29.74 \pm 16.38$ years, DMI prevalence $(86.8 \%)$ were submitted to 72h CGMS (Medtronic; Northridge, CA). Capillary glycemia (CG) and mean CGMS sensor's glycaemic value; correlation coefficient, glycemic excursions (CGMS vs. CG), postprandial hyperglycemia, unrecognized hypoglycemia, complications and therapeutic management after CGMS were analyzed. Alc levels were measured at baseline (I month before) and after 3 months of the study.

RESULTS. The mean capillary glucose values were 191.3 \pm 45.6 vs. $192.5 \pm 43.9 \mathrm{mg} / \mathrm{dl}$ by CGMS sensor, with significant correlation $(p=0.001)$. The correlation coefficient was $0.87 \pm 0.16(V R>0.79)$. The CGMS was significantly more efficient in the detection of glycemic excursion related to capillary glycemia $(p=0.001)$. Postprandial hyperglycemia was identified in $77.3 \%$ of diabetic patients and nocturnal unrecognized hypoglycemia was detected in 54\%. Data showed a significantly decreased Alc level, three months after the CGMS $(p=0.001)$. No complications were registered in $89.8 \%$ of patients.

CONCLUSION. The CGMS was confirmed to bea very safe method, with high accuracy in glycemic values and to have a low complication rate. It is a good method to identify glucose excursion, postprandial hyperglycemia and asymptomatic hypoglycemia, with a significant impact on the Alc levels of diabetic patients. [Rev Assoc Med Bras 2006; 52(6): 395-400]

KEY worDS: CGMS. Capillary glycemia. Diabetes mellitus.

\section{REFERÊNCIAS}

I. Ferraz DP, Maia FFR, Araújo LR. Glicemia capilar em ponta do dedo versus lóbulo de orelha: estudo comparativo dos valores resultantes e preferências dos pacientes. Arq Bras Endocrinol Metab. 2004;48:389-93.

2. Glasgow RE, Eakin EG. Quality of life and associated characteristics in a large national sample of adults with diabetes. Diabetes Care. 1997;20:562-7.

3. Anderson RJ, Freedland KE, Clouse RE, lustman PJ. The prevalence of comorbid depression in adults with diabetes: a meta-analysis. Diabetes Care. $2001 ; 24: 1069-78$.

4. Kaufman FR. Role of continuous glucose monitoring in pediatric patients. Diabetes Technol Ther. 2000;2:S49-52.

5. Boland EA, Tamborlane WW. Continuous glucose monitoring in youth with type 2 diabetes: overcoming barriers to successful treatment. Diabetes Technol Ther 2000;2:S53-9.

6. CaduffA, Hirt E, Feldman Y, Ali Z, Heinemann L. First human experiments with a non-invasive, non-optical continuous glucose monitoring system. Biosens Bioelecton. 2003; 19:209-7.

7. Aussedat B, Dupire-Angel M, Gifford R, Klein JC, Wilson GS, Reach G. Interstitial glucose concentration and glycemia: impiclations for continuous subcutaneous glucose monitoring. Endocrinol Metab. 2000;278:E7|6-E28.

8. Sachedina N, Pickup JC. Performance assessment of the MedtronicMinimed Continuous Glucose Monitoring System and its use for measurement of glycaemic control in Type I diabetic subjects. Diabet Metab. 2003;20: 1012-5.

9. Djakoure-Platonoff C, Radermercker R, Reach G, Slama G, Selam JI. Accuracy of the continuous glucose monitoring system in inpatient and outpatient conditions. Diabetes Metab. 2003;29(2 Pt I): I 59-62.

10. Guerci B, Floriot M, Bohme P, Durain D, Benichou M, Jellimann S, et al. Clinical performance of CGMS in type I diabetic patients treated by continuous subcutaneous insulin infusion using insulin analogs. Diabetes Care. 2003;26: 582-9.

I I. Fiallo-Scharer R. Diabetes Research in Children Network Study Group. Eight point glucose testing versus the continuous glucose monitoring system in evaluation of glycemic control in type I diabetes. J Clin Endocrinol Metab. 2005;90:3387-91 
12. Accuracy of the GlucoWatch G2 Biographer and the continuous glucose monitoring system during hypoglycemia. Experience of the Diabetes Research in Children Network (DirecNet). Diabetes Care. 2004;27:722-6.

13. Ludvigsson J, Hanas R. Continuous subcutaneous glucose monitoring improved metabolic control in pediatric patients with type I diabetes: a controlled crossover study. Pediatrics. 2003; I I I (5 Pt I):933-8.

14. Schaepelynck-Belicar P, Bague P, Simonin G, Lassmann-Vague V. Improved metabolic control in diabetic adolescents using the continuous glucose monitoring system (CGMS). Diabetes Metab. 2003;29:608-12.

15. The Accuracy of the CGMS in children with type I diabetes: results of the Diabetes Research in Children Network (DirecNet). Diabetes Technol Ther. 2003;5:781-9.

16. Steil GM, Rebrin K, Mastrototaro J, Bernaba B, Saad MF. Determination of plasma glucose during rapid glucose excursions with a subcutaneous glucose sensor. Diabetes Technol Ther. 2003;5:27-31.

17. McGowan K, Tomas W, Moran A. Spurius reporting of nocturnal hypoglycemia by CGMS in patients with thigthtyle controlled diabetes. Diabetes Care. 2002;25: I 499-503.

18. Metzger M, Leibowitz G, Waistein J, Glaser B, Raz I. Reproductibility of glucose measurements using the glucose sensor. Diabetes Care. 2002:25: | |85-91.

19. Boland E, Monsod T, Delucia M, Brandt CA, Fernando S, Tamborlane VW. Limitations of conventional methods of self- monitoring of blood glucose: lessons learned from 3 days of continuous glucose sensing in pediatric patients with type I diabetes. Diabetes Care. 2001;24:1 858-62.

20. Chico A, Vidal-Rios P, Subira M, Novials A. The continuous glucose monitoring system is useful for detecting unrecognized hypoglycemias in patients with type 1 and type 2 diabetes but is not better than frequent capillary glucose measurements for improving metabolic control. Diabetes Care. 2003;26:1 I 53-7.

21. Jeha GS, Karaviti PL, Anderson B, Smith EO, Donaldson S, Mc Girk TS, et al. Continuous glucose monitoring and the reality metabolic control in preschool children with type I diabetes. Diabetes Care. 2004;27:288I-6.

22. Maia FFR, Araújo LR. Accuracy, utility and complications of continuous glucose monitoring system (CGMS) in pediatric patients with type I diabetes. J Pediatr. (Rio J) 2005;81:293-7.
Artigo recebido: 02/09/2005

Aceito para publicação: 22/06/2006 